\title{
Discussion of the Analysis of Self-similar Teletraffic with Long-range Dependence (LRD) at the Network Layer Level
}

\author{
G. Millán, H. Kaschel, G. Lefranc
}

\author{
Ginno Millán, Héctor Kaschel \\ Universidad de Santiago de Chile \\ Departamento de Ingeniería Eléctrica \\ Avda. Libertador Bernardo O'Higgins \#3363. \\ Estación Central. Santiago - Chile \\ E-mail: \{ginno.millan,hector.kaschel\}@usach.cl

\section{Gastón Lefranc} \\ Pontificia Universidad Católica de Valparaíso \\ Escuela de Ingeniería Eléctrica \\ Avda. Brasil \#2147. Valparaíso - Chile \\ E-mail: glefranc@ucv.cl
}

\begin{abstract}
Traffic streams, sources as well as aggregated traffic flows, often exhibit long-range-dependent (LRD) properties. This paper presents the theoretical foundations to justify that the behavior of traffic in a high-speed computer network can be modeled from a self-similar perspective by limiting its scope of analysis to the network layer, since the most relevant properties of self-similar processes are consistent for use in the formulation of traffic models when performing this specific task.
\end{abstract}

Keywords: Long-range-dependent, network layer, network traffic, self-similar process.

\section{Introduction}

Still rooted in the genesis of the design of present day high speed computer networks is the trend to scalable development with a base prepared for the primary support of processing applications which, although requiring a reliable transport service, are not demanding in terms of other quality of service (QoS) parameters such as delay, flow rate, latency, and loss rate. It is a reality that is subordinated to financial justifications, unable to reflect both the behavior and the operation of present day network environments, most of them characterized not only by their scalability and support of services and added value applications with high band width and availability requirements, but also by their convergence, complementarity, and interoperability. On the other hand, sustained development in the fields of optical, nanometric, and quantum technologies with greater emphasis has allowed the evolution of computer networks, providing them with the capacity needed to satisfy simultaneously the requirements of diverse traffic, creating the scenario inherent to the appearance of new services and applications for which this characteristic is essential; we are in the presence of services that involve real time traffic and which, because of their nature, have highly demanding needs to and from the available bandwidth. Therefore, the new high speed networks must be capable of providing a service that not only ensures the availability of the resources, but is also provided under quality conditions that are well defined, parameterizable, adaptable, and dynamic in their assignment, because the requirements of present day real time applications and services cannot be satisfied using the high-level protocols if the carrier networks do not offer the necessary guaranties. It is crucial, therefore, to make quantitative analyses that evaluate the service quality offered by the new technologies, leaving 
aside the unsubstantial bases, arguments, and assumptions. The main problem that appears at the time of making a rigorous evaluation of the performance of a communications network is that of modeling the input traffic to the network. In fact, to many authors, traffic modeling is the most critical problem related to the evaluation of the performance of communications networks, because the success of the analyses depends directly on how representative of reality are the traffic models used. Historically, traffic modeling had its origin in conventional telephony systems and has been based almost exclusively on assumptions of independence between the times of arrival of successive grids and exponential durations in the use of the resources. Concretely, the acceptance of both assumptions implies a restriction toward the stochastic processes so that they obey a universe of Poisson or Markov processes. In that respect, the usefulness of their use by network designers as well as by systems analysts for planning capacities and predicting performance is not questioned [1]. However, in a wide range of real world cases it has been verified that the results predicted from the analysis of tails differ significantly from the actual observed performance, and this marked discrepancy has its origin in the fact that traffic processes often present LRD on many or all the temporal scales, while Poisson or Markov models, which have no memory or show short-range dependence (SRD), present traffic flow over much shorter time scales. As a result, there is a tendency to produce highly optimistic forecasts of performance due to the use of distributions with finite variance for characterizing the periods with the presence and absence of burst packets. In view of the above arguments, the following working hypothesis is proposed: "It is completely feasible to restrict the evolution of a statistically self-similar process to a well defined application setting without altering its nature and its more important properties, in that way highlighting the validity of its postulates and giving greater plausibility to its physical interpretation."

In this context, the plausibility refers to the action of conferring an admissible character, therefore worthy of consideration, to one or several parameters that are components of an analytical model, whose interpretations do not constitute only a mathematical idealization. This paper presents a detailed discussion of the theoretical bases that justify the fact that the behavior of a high speed computer network traffic in the presence of long-range dependence can be modeled from a selfsimilar perspective limiting its analysis setting to the network layer level, stressing that all the most relevant properties of the self-similar processes are consistent for use in the formulation of traffic models when this distinction is made, since the need for its concept is justified to describe the traffic that is registered in the settings of present day computer networks.

\section{Bibliographic Discussion}

Since Kleinrock's publication [2], later expanded in [3], which establishes the mathematical theory that governs networks with packet switching, the existence of temporal dependence in the performance of the different types of data traffic flows has become an exciting field of research with countless discoveries, with the huge impact and influence that they have on the performance of tail systems standing out among them. The latter fact accounts for both the current existence and coexistence of a wide variety of input traffic models that show structures with rather complex correlations, which are applied to cases in which the models of the communications systems that are being studied allow adequate analytic handling. In any case, these models, basically Markovian, neglect the temporal correlations starting from a given temporal separation, even if the latter can be increased arbitrarily at the expense of complicating the models with additional parameters. Since 1993 an increasing number of published studies have documented that the data traffic pattern is well modeled by self-similar processes in a wide range of real world and network situations [1], with their top reference point and foundational work in the research of Leland, Willinger, Taqqu, and Wilson [4], presented originally at ACM SIGCOMM '93, and 
then amended and extended in [5]. In spite of the existence of some previous papers that provide informal descriptions of this performance, such as [6]- [8], and to an exception from Mandelbrot himself [9], no one had submitted the idea of self-similarity applied to the analysis of data traffic in itself, and that paper shattered the illusion that a simple tail analysis, based on the assumption that the traffic follows a Poisson distribution can model adequately all the network traffic [1], showing that traffic in Ethernet has a self-similar or fractal nature and therefore requires new modeling and analysis statements. In this respect, the methodology followed by the authors involves a massive compilation of traffic samples from 1898 through 1992, from different Ethernet LANs of the research and engineering center of Bellcore in Morristown, USA [5], which resulted in a detailed temporal high resolution collection totaling more than 100 million packets with $10 \mu$ s precision, grouped in four sets of measures available in [10], and in the application of a rigorous and exhaustive statistical analysis based on the modeling of the traffic sources using hyperbolic tail distributions, in particular Pareto's, comparing the results with the behavior of the traffic flow of the real traces, and in the observation of the estimated value of Hurst's parameter $(\mathrm{H})$ for each of the four sets of traffic samples, expressed for processing as a series of ordered pairs of data composed of the time of arrival and the size of the Ethernet packet, as well as for each level of temporal traffic aggregation considered.

A complete analysis of both the statement and the methodology followed by the authors is found in [11] and [12], and their proofs in [13]. Specifically, this research shows that:

- It is possible to model Ethernet traffic producing results similar to those of real Ethernet traffic using few parameters (parsimony), and with the fundamental added value of being physically plausible.

- Ethernet LAN traffic can be modeled through the superposition of many sources that vary between a state of burst transmission and one of inactivity, using for their characterizations infinite variance distributions. In particular it is proved using a Pareto distribution.

- Ethernet traffic is statistically self-similar regardless of the place and time at which it is checked.

- The degree of self-similarity measured in terms of Hurst's parameter $(\mathrm{H})$ is a function of the use factor of Ethernet and can be used to get the magnitude of the traffic bursts.

- Traditional traffic models are unable to capture the property of self-similarity.

These results and their deep implications, as can be noted from the preceding paragraphs, produced a host of researchers seeking to observe that same behavior associated with the largest variety of communications and applications scenarios.

What follows is an exhaustive literature review of research results in relation to their field of application, that approaches the treatment of systems communications systems and applications from a self-similar perspective. It should be noted that the idea is not only to show the application of this view, but also to present results that dissent from it. The self-similar or fractal behavior of traffic in WAN networks is shown in [14]- [16], pointing out the failure of Poisson models to represent the strong correlations that exist at different temporal scales. Evidence and conclusions on this behavior in traffic due to the WWW are provided in [17]- [19], considering interconnection scenarios as well as traffic patterns in browsers. On the other hand, [20] and [21] point out the fractal nature of the data flow of the protocols that compose the signaling system 7 in common channel signaling networks [22], showing that the traditional methods are not adequate to interpret their behavior, and the duration of the calls are better characterized if hyperbolic tail distributions are used. In another setting, [9] and [23]- [25] show that the LRD 
is a characteristic inherent to VBR video traffic that does not have any relation with the type of codec or the number of special effects that contain the recorded scenes. Specifically, the VBR video traffic flow transmitted through B-ISDN, ATM, and Internet networks are studied, showing that the behavior of the distribution's tail that represents the marginal band width can be described exactly if hyperbolic tail distributions (like Pareto) are used; that the self-correlation function of the video sequences decays hyperbolically (this is equivalent to long-range dependence) and it can be modeled using self-similar processes, and finally, that the use of models that only capture the SRD is inappropriate for characterizing this kind of traffic because it overestimates the performance, leading to insufficient resource assignments, which finally derives in poor perceptions by the users of the networks when difficulties appear to achieve the service quality expected by them. In the field of wireless communications, in [26] it is shown that traffic in CDPD networks has an LRD behavior. Using the R/S and Variance-Time methods, it estimates values for Hurst's parameters of $\mathrm{H}=0.8$ and $\mathrm{H}=0.9$, respectively, thus disregarding the use of predictive models based on Poisson arrival processes. In [27] an investigation is made of the impact of mobility on the added traffic in wireless networks in the city of Bristol, UK, and of whether the applications of voice and data together produce self-similar traffic. It is concluded that the added traffic generated by the mobile users that use voice and data services together show a self-similar LRD behavior that has no relation with the rate of penetration of the services. They warn on the drastic changes that wireless multimedia service implementations must undergo in terms of the traffic profiles used in their models to be able to capture that characteristic. From another perspective, in [28] it is shown that traffic in wireless networks with ad hoc topology is self-similar and forecastable as a consequence of the fact that subjacent temporal self-similar series are, in essence, predictable. The required data are captured using a wireless test network with ad hoc topology. The analysis of traffic and the design of wireless IP networks describing TCP traffic as dominant in present day Internet is approached in [29], indicating that its statistical nature shows the same behavior over all the temporal scales. It also presents an analysis of traffic traces which shows the statistically self-similar nature of the traffic due to WWW and to the VBR video over these types of networks. In [30] develops a new model for traffic in wireless networks that has its origin in the alternating fractal renewal processes (AFRP) proposed as traffic models in [14], and in the wide band network traffic model using the extended alternating fractal renewal processes (EAFRP) proposed in [31]. With the incorporation of a limiting rate for alternation between the two states, called extended limiting rate, the Rate-Limited EAFRP model is formulated, which assumes an advance with respect to the wireline models used traditionally for model traffic in wireless networks, since it takes care of its two main deficiencies: omission of the effects of the LRD temporal correlations, and inability to make reliable performance forecasts due to the high dependence on short-range processes. In [32] there is an extensive discussion of the problem of modeling the data traffic that flows from and to the wireless networks with respect to the Internet, taking care of large scale wireless communication structures. Based on the methodology presented in [33] and tested extensively in [34], [35], it is concluded that the circulation of traffic flows cannot be treated using Poisson models, and that their behavior is statistically self-similar. In [36] two solutions are proposed to create an interconnecting bridge between WiMAX and WiFi links. The former is based on maintaining a certain level of QoS from one end to the other regardless of the wireless technology used, while the latter is aimed at the reduction of the complexity in its physical implementation at the expense of not providing any QoS guaranty. In both cases the performance of the system is compared with computer simulations that consider real time traffic with long-range dependence that is manifested trough a polynomial type decay of the self-correlation function. To model the traffic generated by the many terminals within the WLAN, recourse is made to the On/Off methodology presented in [14], supporting its foundations on [37]. Finally, in the same context, 
but in another field of application, [38] deals with the use of fractal geometry in the antenna design process, while in [39] a general synthesis methodology is proposed that covers the efficient design of fractal antennas and their WiMAX applications. In the field of optical networks, [40] deals extensively with the use of self-similar traffic models, in particular that proposed in [15], as the only way to represent reality faithfully and to evaluate the performance of Ethernet passive optical networks. In [41] a new protocol is proposed for labeled switching in optical networks with optical burst switching (OBS), with time/space labeling that allows to keep the signaling always joined with the addressing functions. The results obtained are based on simulations under the premise of accepting the self-similarity of the traffic in the networks with wavelength division multiplexing. Finally, [42] reports on the implications of reducing the self-similarity of IP traffic by the OBS assembly algorithms. In spite of what all the above arguments imply in terms of putting in evidence the merits and advantages of the use of parsimonious models and which also provide a plausible physical interpretation to its parameters, the question arises on the degree of prevalence of these self-similar traffic patterns and on which are the conditions for the analysis of performance to depend critically on considering self-similarity. In this sense it is valid to inquire not only about the origin of the data that have been analyzed with respect to the synthetic traces generated, but also on what is the context or the setting in which these comparisons have been made, and where are the results aimed at. It is no less true that in the light of all the research presented, it seems even irrelevant to think of a traditional analysis of tails to represent data traffic flow in present day high speed networks. But this is neither categorical nor restrictive; this methodology cannot be disregarded flatly, and neither is its proper to think that the former is the unified solution by simply arguing it ubiquitousness in all the temporal scales, because this, analyzed from a higher perspective, and even though the presence of the correlation in the traffic is not under discussion, brings about the question of whether the correlation structure alone is sufficient to characterize traffic over self-similar processes. The context described in this way is very extensive, and therefore the results must be limited.

There are several reports that put in evidence the lack of consensus on the application field of self-similar models and of the impact of LRD on the performance of communications systems, and even though their number is much more limited than that of those whose results support exactly the opposite, their conclusions must be analyzed carefully because they bring forth, in essence, a critical and fundamental matter in common: "since the traditional tail models are unable to put in evidence the self-similarity characteristic, its validity for predicting performance would be supported if it is shown that self-similarity does not have a measurable impact on performance," and even more so if it is shown that the models based on self-similar stochastic processes fail when considering the impact of important characteristic parameters in each particular network case. Precisely based on this last point, [43] presents a detailed analysis of a fault detected in self-similar models by considering that they are incapable of reflecting the impact of the range of temporal scales of interest for evaluating the performance and the prediction of problems, and those first order statistics such as the marginal distribution of the process. Based on traces generated by a JPEG encoder of an NTSC television channel and the traces of [6] it is reported that:

- There is a correlation horizon such that the rate of loss does not affect performance beyond it.

- The correlation level considered for evaluating performance depends not only on the structure of the source traffic correlation, but also on the temporal scales belonging to the system that is being studied.

- The scale factor considered has a considerably greater impact on the rate of loss than Hurst's parameter or the size of the buffers. 
- Increasing the size of the buffers helps reduce the rate of loss if we are dealing with SRD traffic. On LRD traffic it does not have a considerable impact.

The in-depth analysis of this work shows that the impact of the LRD is dealt with over the contribution of the single server model in relation to the size of the input buffer, using for that purpose a model of fluid [44], [45], that presents a hyperbolic drop down to a given cut coefficient from which it drops to zero. Based on the results from many simulation experiments using video traces as well as Ethernet traces for different values of Hurst's parameter, cut coefficients and buffer sizes, and a wide range of marginal distributions, the authors discovered the existence of a critical cut coefficient that they call "correlation horizon," such that the rate of loss is not affected if the cut coefficient is increased above it. Therefore, the correlation horizon separates the relevant from the irrelevant correlation coefficients with respect to the rate of loss. Finally, in their conclusions the authors argue that because of the existence of a finite cut horizon (which in the case of the finite buffers is a function of their size), any model that captures the correlation structure up to that horizon will be valid to represent the system. On the contrary, if the correlation horizon is infinite, i.e., the system's time scale cannot be determined clearly, then selfsimilar models must be used. Based on the above point, [46] states that in the case of considering a finite buffer, the effects of the LRD are detectable only if it makes the occupation periods become sufficiently long, since the behavior of their tail is affected largely by the characteristics of the traffic that arrives during those periods. In that respect, this appraisal is based on [47], where through the definition of the concept of "relevant temporal scale" as the typical duration at which all the arrivals at a tail interact and affect collectively their behavior, it is deduced that for the large buffers the size of the tail can be large, so many arrivals interact in the tail and cause the long-range correlation to cause more losses than those predicted by the models that are not capable of considering it or do not consider it. However, in the case of small buffers, where few arrivals interact, the effect of the LRD is imperceptible. Therefore, the standpoint on the appraisal of the effects of the LRD in terms of its impact on the occupied periods is depicted by the authors through the concept of "reset effect," which involves that as the buffer in question is emptied, the system forgets everything. In this way, in the case of VBR video servers, since they are sensitive to the delay and to the loss of meshes, the intensity of the traffic flow will not be very large, giving rise to short periods of occupation and a very pronounced reset effect in the regions of practical operation, an effect that will also be reinforced in the case of finite buffers, due to the truncating effect of these types of buffers. The latter is due to the fact that an occupied period in which there is overflow is shorter than the corresponding one in an infinite buffer model, or similarly, there can be several periods occupied in the finite buffer version before the corresponding occupation period ends in the infinite buffer version. Then, through the use of Markov models, the authors finally report that when the SRD is strong and parameter $\mathrm{H}$ is moderate, the LRD has no impact on the occupation of the buffer, and therefore their models give rise to good estimations, and in the case in which the SRD shows a slightly pronounced behavior and parameter $\mathrm{H}$ has a high value, the truncating effect is sufficiently strong for their models to estimate well the rate of loss, even though it is admitted that for a high traffic intensity and a large buffer size the estimation of the mean size of the tail is bad. In [48], a research having characteristics similar to those of the previous one, the concept of critical temporal scale (CTS) is introduced as follows. Given the size of the buffer and the marginal distribution of mesh sizes, the CTS of a VBR video source is defined as the number of mesh correlations that contribute effectively to the rate of loss of cells. Using models of the video traces of [9], the authors state that for Markovian models as well as with LRD, the CTS is finite and decreases with the size of the buffer. Consequently, and under the assumption that the size of the buffer required to multiplex a large number of VBR video source is typically small due to the restrictions that correspond to real time applications, it is concluded that for buffer sizing scenarios in ATM 
networks it is not necessary to capture the correlations with the LRD of a video source even if the traffic shows this behavior markedly, because for all practical cases the SRD has a dominant impact. The following can be argued with respect to this last conclusion and to the research in general:

- The result is based on an analysis that relies completely on marginal Gaussian distributions, and even though it can be explained from the perspective of keeping an adequate analytic treatment, it is not sufficient for validating absolute generalizations of the "in all cases" type nor to attenuate the fact of working with distributions independent of the behavior of others.

- The behavior of the models if hyperbolic tail distributions are used or if they are tested with distributions without margins is not reported.

An extended version of this research is [49], where not only these two considerations are taken up, but attention is also given to the relevance of the SRD and the LRD in real time VBR video traffic in wide band networks (particularly ATM) and in the integrated Internet services, and through a theoretical and simulations approach, the authors tackle the problem of determining admissible ranges for the probability of cell loss and its relation with the size of the buffers in terms of the maximum delay. It should be noted that this research takes place within the context of validating the use of Markovian models and SRD for video applications, and for that purpose the authors show basically that:

- The long-range correlations have no impact on the probability of cell loss.

- An adequately implemented Markov model that captures the relevant range of correlation provides good predictions of performance.

- The capture of the LRD by itself can lead to an underestimation of the necessary resources in the network.

- The CTS explains the strong relation existing between the probability of cell loss in ATM and the SRD at the expense of the LRD. That is, for the applications of interest, the CTS is small and more sensitive to the short-range than to the long-range traffic correlations.

- Analytically simple Markovian models are feasible and have the capacity to harmonize marginal distributions and correlations over a given critical temporal scale.

As a last example of the already mentioned lack of consensus in the field of video traffic, [61] presents a model of a VBR traffic source that uses finite states Markov chains, and states that although the original model presented in [26] is good in terms of its parsimony, it does not lend itself adequately to analytic studies. Summarizing, six reports have been presented so far with a common denominator: dissenting on the blind applicability of the self-similar model and its implications in performance, considering as examples systems that involve video traffic, mainly of the VBR type. But as expected, this is not the only field in which discrepancies appear, and more acutely and in direct relation with the working hypothesis stated there is a number of reports that question the validity of the use of Hurst's parameter as a single descriptor to characterize the LRD of a self-similar stochastic process. But before dealing with this topic a last group of reports are mentioned that have a critical position toward self-similar models in areas other than video traffic. In the field of wireless networks and their associated technologies, [51] studies the behavior of the self-similarity characteristic of traffic when it goes from a wired to a wireless network through a gateway, concluding that the device can change the traffic's degree of selfsimilarity as a direct consequence of the reassembly and repacking operations on the self-similar 
input traffic, even reaching its annulment. In [52] the above behavior is reaffirmed considering the study of the influence of the MAC mechanism of IEEE 802.3e on LRD traffic when it goes through one or several links, suggesting that the traffic transported through a WLAN interface undergoes deep structural changes in its statistical model, and showing that the fractional Gaussian traffic model is inadequate to describe its behavior. Finally, in the field of OBS networks, [53] reports the development of an algorithm for the assembly of bursts that has the purpose of reducing the degree of self-similarity of IP traffic. It is admitted that this is a characteristic inherent to WWW traffic, but its presence causes an important disadvantage in terms of the performance of the tails, so it must be reduced in favor of a random SRD traffic. The first paragraph of this page mentions the existence of some research that questions the use of Hurst's parameter as a single indicator to capture the self-similarity characteristic of a stochastic process that boasts of being such, in addition to stressing the importance that this fact has to prove the stated work hypothesis. In this respect, and recalling its statement, it is specified that the idea is not to validate exhaustively the self-similarity parameter or Hurst's parameter, but only to obtain an indicator of the presence of the characteristic in representative traffic series. Consider the following definition: The valuated real process $X(t), t \in \mathbb{R}$ is self-similar with $H>0$ if for all $a>0$ the finite-dimensional distributions of $X(a t), t \in \mathbb{R}$ are identical to the finite-dimensional distributions $a^{H} X(t), t \in \mathbb{R}$, i.e.,

$$
\{X(a t), t \in \mathbb{R}\}={ }_{d}\left\{a^{H} X(t), t \in \mathbb{R}\right\} \quad \forall a>0
$$

where $=_{\mathrm{d}}$ means equality for all the finite-dimensional distributions [54], [55].

The property defined by (1), is usually known as the scaling property, and a direct consequence of its definition is that a self-similar process preserves its distribution, and thereby its statistics, since it is subjected to a temporal scaling. Also, from the same standpoint parameter H, or Hurst's, is known as the self-similarity parameter for the stochastic process $X(t)$ to which it is associated. The first report that recognized and approached the need to have additional parameters to characterize the variability of the traffic is [26], where although it is accepted that $\mathrm{H}$ is necessary for that purpose, it is not sufficient. Through a detailed statistical analysis of VBR video samples, the authors conclude that the self-correlation of the VBR video sequences decays hyperbolically, equivalent to the LRD. But since the LRD is related to the frequency of the components of the process and not to the distribution of the bandwidth requirements, if the marginal distribution is compressed because the coefficient of variability (coefficient between the mean bandwidth and the standard deviation) tends to zero when the number of multiplexed input sources that give rise to the traffic tend to infinity, the traffic, as the number of sources increases, is confined within narrow statistical limits, and although within these frontiers the behavior continues to be long range (result confirmed through $\mathrm{H}=0.7$ ), in the range in which the standard deviation is much less than the product of the mean of the bandwidth distribution and the number of multiplexed input sources, the traffic does not depend on $\mathrm{H}$. Therefore, $\mathrm{H}$ is necessary to characterize the variability, but it is not sufficient. So an adequate characterization of video traffic must consider at least the following four parameters: the mean of the bandwidth distribution, the number of multiplexed input sources, the standard deviation, and the coefficient of variability derived from them. The authors make it clear, finally, that these results are valid only if they follow the central limit theorem [56], i.e., when the standard deviation is finite. Although the above result may seem to be expected because the parsimony can lead to imprecision in both the interpretations and the results, it is not so in relation to the effect produced by assuming that if the detailed behavior of the components of a given stochastic process, P1, that shows some degree of self-similarity $\mathrm{H} 1$ is not known, then a biunivocal correspondence between $\mathrm{H} 1$ and P1 is clearly established. In other words, processes that show clearly differentiated behaviors are possible, but their correlation structures must be characterized by the same $\mathrm{H}$ parameter. In this 
respect, [57] approaches this problem considering the asymptotic behavior of an unlimited buffer of a multiplexor under different self-similar input traffic models, in particular, Cox's infinite server models, or $\mathrm{M} / \mathrm{G} / \infty$ [58], and fractional Gaussian noise models [59], [60]. In this way the authors report getting two completely different behaviors for the buffer's probability tail, namely that while with the former the drop is mostly hyperbolic, with the latter it presents a Wiebull asymptotic behavior [61], simply showing that Hurst's parameter is insufficient as a single descriptor to characterize the LRD in input traffic models [62], [63]. In [64] it is also shown how synthetic traces that have identical self-similarity parameters and means differ significantly from one another. Finally, in [65] the insufficiency of Hurst's parameter by itself as a precise descriptor of the long-range dependence of the traffic in an Ethernet network is reported. In this research it is shown convincingly, through an analysis of tails applied to a series of representative data of the traces of the real traffic registered in the Ethernet network of the Department of Computer Science of the University of California at Los Angeles, CA, USA, that Hurst's parameter does not provide a precise prediction of the performance of the tails for a given LRD traffic, and that its behavior is not monotonic with respect to the presence or absence of bursts if the original series is disaggregated into smaller ones, which also implies that $\mathrm{H}$ does not serve to characterize the relative importance of groups within a whole. It is clearly seen that both results are opposed to the conventionalism of [6] since $\mathrm{H}$ cannot be used to size the traffic bursts in Ethernet. Questions on where, when, and under what set of circumstances the use of self-similar processes in modeling communications systems and related applications is completely valid, as well as the uncertainty on the existence of a scenario that brings together criteria and its influence on the basic characteristics of the processes remain unanswered. However, they seem to have an answer derived from the analysis of the research of Ryu and Lowen, [66], [67], on the use of fractal point processes (FPP) for modeling and analyzing self-similar traffic in networks [68]. Concretely, this research proposes to make a distinction between self-similarity at the application level and self-similarity at the network level for the purpose of the design and administration of wideband networks in terms of a correct provision of the QoS required by the applications, and their best results are the proofs that self-similarity of VBR traffic can frequently be ignored in the face of the sizing of the buffers in ATM, and that self-similar traffic at the applications level can be managed effectively in the context of the admission control for assigning resources with service quality guarantees, because it is independent of the network conditions under which it is sent.

\section{Conclusions}

A detailed discussion has been presented of the theoretical bases that support the position of considering that high speed computer network traffic shows self-similar behavior and long-range dependence. With respect to the above, and considering the arguments in favor and against this position, it is believed that traffic in present day computer network settings is of a statistically self-similar nature and presents a pronounced long range dependence (LRD). Accepting the above singularities as inherent to the traffic flow of present day high speed network settings, it is proposed that their behaviors are amenable to being modeled by limiting their applicability to the network layer level, estimating that the most relevant properties of self-similar stochastic processes are consistent to be used in the formulation of traffic models when that distinction is made, since the concepts of self-similarity and long-range dependence are justified by the need to describe faithfully the real traffic processes in present day computer network settings. To depict the concepts with which this research deals, the following work hypothesis is proposed:

"It is completely feasible to restrict the evolution of a statistically self-similar process to a well defined application setting without altering its nature and its more important properties, in that 
way highlighting the validity of its postulates and giving greater plausibility to its physical interpretation."

Considering all the arguments given above, it is stated that it is theoretically feasible to prove it, since all its arguments are correctly founded and supported, and only their proof at the analytic and experimental levels remains as a future task.

\section{Bibliography}

[1] W. Stallings, Redes e Internet de Alta Velocidad. Rendimiento y Calidad de Servicio, 2nd ed., Madrid, Pearson - Prentice Hall, 2004, pp. 224-237.

[2] Kleinrock, Information Flow in Large Communication Nets, Ph.D. Thesis, Research Laboratory of Electronics, Massachusetts Institute of Technology, Cambridge, MA, 1961.

[3] L. Kleinrock, Communication Nets: Stochastic Message Flow and Delay, New York, McGrawHill, 1964.

[4] W.E. Leland, M.S. Taqqu, W. Willinger, and D.V. Wilson, On the Self-Similar Nature of Ethernet Traffic, in Proc. ACM SIGCOMM ?93, San Francisco, CA, pp. 183-193.

[5] W.E. Leland, M.S. Taqqu, W. Willinger, and D.V. Wilson, On the Self-Similar Nature of Ethernet Traffic (Extended Version), IEEE/ACM Trans. Netw., Vol. 2, No. 1, pp. 1-15, February 1994.

[6] A. Erramilli, R.P. Singh, and P. Pruthi, Application of Deterministic Chaotic Maps to Model Packet Traffic in Broadband Networks, in Proc. 7th ITC Specialist Seminar, Morristown, NJ, 1990.

[7] W.E. Leland and D.V. Wilson, High Time-Resolution Measurement and Analysis of LAN Traffic: Implications for LAN Interconnections, in Proc. IEEE INFOCOM ?91, Bal Harbour, FL, pp. 1360-1361.

[8] J. Beran, R. Sherman, M.S. Taqqu, and W. Willinger, Long-Range Dependence in VariableBit-Rate Video Traffic, IEEE Trans. Commun., Vol. 43, No 2/3/4, pp. 1566-1579, Feb/Mar/Apr 1995.

[9] B. Mandelbrot, Self-Similar Error Cluster in Communication Systems and the Concept of Conditional Stationarity, IEEE Trans. Commun. Technol., Vol. 13, No. 1, pp. 71-90, Mar. 1965.

[10] http://ita.ee.lbl.gov/html/contrib/BC.html.

[11] W. Willinger, M.S. Taqqu, R. Sherman, and D.V. Wilson, Self-Similarity Through HighVariability: Statistical Analysis of Ethernet LAN Traffic at the Source Level, in Proc. ACM SIGCOMM '95, Cambridge, MA, pp. 100-113.

[12] W. Willinger, M.S. Taqqu, R. Sherman, and D.V. Wilson, Self-Similarity Through HighVariability: Statistical Analysis of Ethernet LAN Traffic at the Source Level, IEEE/ACM Trans. Netw., Vol. 5, No. 1, pp. 71-86, Feb. 1997.

[13] M.S. Taqqu, W. Willinger, and R. Sherman, Proof of a Fundamental Result in Self-Similar Traffic Modeling, ACM SIGCOMM Computer Communication Review, Vol. 27, No. 2, pp. 5-23, Apr. 1997. 
[14] A. Adas and A. Mukherjee. (1994, Dec.). On Resource Management and QoS Guarantees for Long Range Dependent Traffic. Georgia Inst. Tech., GA. [Online]. Available: http://hdl.handle.net/1853/6797.

[15] S.M. Klivansky and A. Mukherjee. (1995, Aug.). The NFSNET. Georgia Inst. Tech., GA. [Online]. Available: ftp://ftp.cc.gatech.edu/pub/coc/tech_reports/95/GIT-CC-95-07.ps.Z.

[16] V. Paxson and S. Floyd, Wide-Area Traffic: The Failure of Poisson Modeling, IEEE/ACM Trans. Netw., Vol. 3, No. 3, pp. 226-244, Jun. 1995.

[17] M.E. Crovella and A. Bestavros. (1995, Oct.). Explaining World Wide Web Traffic SelfSimilarity. Boston Univ., MA. [Online]. Available: http://www.cs.bu.edu/techreports.

[18] M.E. Crovella and A. Bestavros, Self-Similarity in World Wide Web Traffic: Evidence and Possible Causes, IEEE/ACM Trans. Netw., Vol. 5, No. 6, pp. 835-846. Dec. 1997.

[19] M. Arlitt, R. Friedrich, and T. Jin, Workload Characterization of a Web Proxy in a Cable Model Environments, Performance Evaluation Review, Vol. 27, No. 2, pp. 25-36, Sep. 1999.

[20] D.E. Duffy, A.A. Mc Intosh, M. Rosenstein, and W. Willinger, Statistical Analysis of CCSN/SS7 Traffic Data from Working CCS Subnetworks, IEEE J. Sel. Areas Commun., Vol. 12, No. 3, pp. 544-551, Apr. 1994.

[21] P. Pruthi and A. Erramilli, Heavy-Tailed ON/OFF Source Behavior and Self-Similar Traffic, in Proc. 1995 IEEE International Conference on Communications, Seattle, WA, Vol. 1, pp. 445-450.

[22] G. Rufa, Developments in Telecommunications. Whit a Focus on SS7 Network Reliability, Berlin, Germany: Springer-Verlag, 2008.

[23] M.W. Garrett and W. Willinger, Analysis, Modeling and Generation of Self-Similar VBR Video Traffic, Computer Communication Review, Vol. 24, No. 4, pp. 269-280, Oct. 1994.

[24] B. Tsybakov and N.D. Georganas, On Self-Similar Traffic in ATM Queues: Definitions, Overflow Probability Bound and Cell Delay Distribution, IEEE/ACM Trans. Netw., Vol. 5, No. 3, pp. 397-409, Jun. 1997.

[25] L. Yellanki, Performance Evaluation of VBR Video Traffic Models, M.Sc. Thesis, Dept. Comput. Sci., Univ. Saskatchewan, Saskatoon, SK, Canada, 1999.

[26] M. Zhonghua, Analysis of Wireless Data Network Traffic, M.Sc. Thesis, School of Engineering Science, Simon Fraser Univ., Burnaby, BC, Canada, 2000.

[27] D.R. Basgeet, J. Irvine, A. Munro, P. Dugenie, D. Kaleshi, and O. Lazaro, Impact of Mobility on Aggregate Traffic in Mobile Multimedia System, in the 5th International Symposium on Wireless Personal Multimedia Communications, Honolulu, HI, 2002, Vol. 2, pp. 333-337.

[28] Q. Liang, Ad Hoc Wireless Network Traffic-Self-Similar and Forecasting, IEEE Commun. Lett., Vol. 6, No. 7, pp. 297-299, Jul. 2002.

[29] T. Janevski, Characterization and classification of IP traffic, in Traffic Analysis and Design of Wireless IP Networks, Norwood, MA: Artech House, Inc., 2002, ch. 5, pp. 135-165.

[30] J. Yu, Modeling of High-Speed Wireline and Wireless Network Traffic, Ph.D. Dissertation, Elect. Comput. Eng. Dept., Drexel Univ., Philadelphia, PA, 2005. 
[31] X. Yang, Impulsive Self-Similar Processes, with Applications in Broadband Communication System Modeling, Ph.D. Dissertation, Elect. Comput. Eng. Dept., Drexel Univ., Philadelphia, PA, 2001.

[32] J. Ridoux, A. Nucci, and D. Veitch, Seeing the Difference in IP Traffic: Wireless Versus Wireline, in Proc. 25th IEEE International Conference on Computer Communications, Barcelona, Spain, 2006, pp. 1-12.

[33] N. Hohn, D. Veitch, and P. Abry, Does Fractal Scaling at the IP Level Depend on TCP Flow Arrival Processes?, in Proc. 2nd ACM SIGCOMM Workshop on Internet Measurement, Marseille, 2002, pp. 63-68.

[34] N. Hohn, D. Veitch, and P. Abry, Cluster Processes, a Natural Language for Network Traffic, IEEE Trans. Signal Process., Vol. 51, No. 8, pp. 2229-2244, Aug. 2003.

[35] N. Hohn, D. Veitch, and P. Abry, The Impact of the Flow Arrival Process in Internet Traffic, in Proc. IEEE Int. Conf. Acoustics, Speech, and Signal Processing, Hong Kong, 2003, Vol. 6, pp. VI-37-40.

[36] R. Fantacci and D. Tarchi, Bridging Solutions for a Heterogeneous WiMAX-WiFi Scenario, Journal of Communications and Networks, Vol. 8, No. 4, pp. 369-377, Dec. 2006.

[37] A. Erramilli, M. Roughan, D. Veitch, and W. Willinger, Self-Similar Traffic and Network Dynamics, in Proc. of the IEEE, Vol. 90, No. 5, pp. 800-819, May. 2002.

[38] J.P. Gianvittorio and Y. Rahmat-Samil, Fractal Antennas: A Novel Antenna Miniaturization Technique, and Applications, IEEE Antennas Propagat. Mag., Vol. 44, No. 1, pp. 20-36, Feb. 2002.

[39] R. Azaro, E. Zeni, M. Donelli, and A. Massa, Fractal-based methodologies for WiMAX antenna synthesis, in WiMAX: Technologies, Performance Analysis, and QoS, S. Ahson and M. Ilyas, Eds. Boca Raton, FL: CRC Press, 2008, ch. 2, pp. 21-39.

[40] G. Kramer, Ethernet Passive Optical Networks, USA, McGraw-Hill, 2005.

[41] A. Huang, B. Mukherjee, L. Xie, and Z. Li, Time-Space Label Switching Protocol (TSL$S P)$, in High-Performance Packet Switching Architectures, I. Elhanany and M. Hamdi, Eds., Germany: Springer-Verlag, 2007, ch. 9, pp. 197-210.

[42] M. Maier, Optical Switching Networks, New York: Cambridge University Press, 2008.

[43] M. Grossglauser and J-C Bolot, On the Relevance of Long-Range Dependence in Network Traffic, IEEE/ACM Trans. Netw., vol. 7, no. 5, pp. 629-640, Oct. 1999.

[44] J.-Y. Le Boudec and P. Thiran, Network Calculus. A Theory of Deterministic Queuing Systems for the Internet, Germany: Springer-Verlag, 2004, pp. 3-6.

[45] A. Adas, Traffic Models in Broadband Network, IEEE Commun. Mag., Vol. 35, No. 7, pp. 82-89, Jul. 1997.

[46] D.P Heyman and T.V. Lakshman, What are the Implications of Long-Range Dependence for VBR-Video Traffic Engineering?, IEEE/ACM Trans. Netw., Vol. 4, No. 3, pp. 301-317, Jun. 1996. 
[47] K. Sriram and W. Whitt, Characterizing Superposition Arrival Processes in Packet Multiplexers for Voice and Data, IEEE J. Sel. Areas Commun., Vol. 4, No. 6, pp. 833-846, Sep. 1986.

[48] B.K. Ryu and A. Elwaid, The Importance of Long-Range Dependence of VBR Video Traffic in ATM Traffic Engineering: Myths and Realities, Computer Communication Review, vol. 26, no. 4, pp. 3-14, Oct. 1996.

[49] B.K Ryu and A. Elwaid, The Relevance of Short Range and Long-Range Dependence of VBR Video Traffic to Real-Time Traffic Engineering, unpublished.

[50] K. Chandra and A.R. Reibman, Modeling One-and Two-Layer Variable Bit Rate Video, IEEE/ACM Trans. Netw., Vol. 7, No. 3, pp. 398-417, Jun. 1999.

[51] J. Yu and A. Petropulu, Is High-Speed Wireless Network Traffic Self-Similar?, in Proc. of IEEE Int. Conf. Acoustics, Speech, and Signal Processing, Montreal, Canada, 2004, Vol. 2, pp. II-425-428.

[52] S. Bregni, P. Giacomazzi, and G. Saddemi, Transport of Long-Range Dependent Traffic in Single-Hop and Multi-Hop IEEE 802.3e Networks, in Proc. IEEE Global Telecommunications Conference, New Orleans, LA, 2008, pp. 1-6.

[53] A. Ge, F. Callegati, and L.S. Tamil, On Optical Burst Switching and Self-Similar Traffic, IEEE Commun. Lett., Vol. 4, No. 3, pp. 98-100, Mar. 2000.

[54] D.I. Sheluhin, S.M. Smolskiy, and A.V. Osin, Self-Similar Processes in Telecommunications, Chichester, UK: John Wiley \& Sons, Ltd., 2007, ch.1, pp. 8-9.

[55] X. Yang, Impulsive Self-Similar Processes, with Applications in Broadband Communication System Modeling, Ph.D. Dissertation, Elec. Comput. Eng. Dept., Drexel Univ., Philadelphia, PA, 2001.

[56] F. M. Dekking, C. Kraaikamp H. P. Lopuhaä, and L. E. Meester, The central limit theorem, in A Modern Introduction to Probability and Statistics. Understanding Why and Wow. Springer-Verlag, 2005, ch. 14, pp. 195-202.

[57] M. Parulekar and A.M. Makowski, Tail Probabilities for a Multiplexer with Self-Similar Traffic, in Fifteenth Annual Joint Conf. IEEE Computer Societies. Networking the Next generation, San Francisco, CA, 1996, vol. 3, pp. 1452-1459.

[58] M. Parulekar and A.M. Makowski. (1996). M/G/ $\infty$ Input Process: A Versatile Class of Models for Network Traffic, Univ. Maryland, College Park, MD, [Online]. Available: http://hdl.handle.net/1903/5778.

[59] B. Mandelbrot and W. Van Ness, Fractional Brownian Motions, Fractional Noises and Applications, SIAM Review, Vol. 10, No. 4, pp. 422-437, Oct. 1968.

[60] J. Beran, Statistical Methods for Data with Long-Range Dependence, Statistical Science, Vol. 7, No. 4, pp. 404-416, Nov. 1992.

[61] H. Rinne, The Weibull Distribution. A Handbook, Boca Raton, FL: Chapman \& Hall/CRC, 2009. 
[62] M. Parulekar and A.M. Makowski. (1995). Buffer Overflow Probabilities for a Multiplexer with Self-Similar Traffic. Univ. Maryland. College Park, MD, [Online]. Available: http://hdl.handle.net/1903/5727.

[63] M. Parulekar and A.M. Makowski (1996). Tail Probabilities for $M|G| \infty$ Input Processes (I): Preliminary Asymtotics. Univ. Maryland. College Park, MD, [Online]. Available: http://hdl.handle.net/1903/5760.

[64] A. Patel and C. Williamson, Statistical Multiplexing of Self-Similar Traffic: Theoretical and Simulation Results, unpublished.

[65] R. Ritke, X. Hong, and M. Gerla, Contradictory Relationship Between Hurst Parameter and Queuing Performance (extended version), Telecommunication Systems, Vol. 16, No. 1-2, pp. 159-175, Jan. 2001.

[66] B.K. Ryu and S.B. Lowen, Point Process Approaches for Modeling and Analysis of SelfSimilar Traffic: Part I: Model Construction, in Proc. Fifteenth Annual Joint Conference of the IEEE Computer Societies. Networking the Next Generation, San Francisco, CA, 1996, vol. 3, pp. 1468-1475.

[67] B.K. Ryu and S.B. Lowen, Point Process Approaches for Modeling and Analysis of SelfSimilar Traffic: Part II: Applications, in Proc. 5th International Conference on Telecommunication Systems, Modeling, and Analysis, Nashville, TN, 1997, pp. 62-70.

[68] S.B. Lowen and M.C. Teich, Estimation and Simulation of Fractal Stochastic Point Processes, Fractals. Complex Geometry Patterns and Scaling in Nature and Society, vol. 3, no. 1, pp. 183-210, Mar. 1995. 